\title{
Synthesis and spectroscopy of benzylamine-substituted BODIPYs for bioimaging
}

\author{
Consuelo Ripoll ${ }^{[a]}$, Cheng Cheng, ${ }^{[b]}$ Emilio Garcia-Fernandez, ${ }^{[a]} \mathrm{Jin} \mathrm{Li},{ }^{[b]}$ Angel Orte, ${ }^{[a]}$ Hainam Do, ${ }^{[c]}$ \\ Lijuan Jiao, ${ }^{* b]}$ David Robinson, ${ }^{[\mathrm{d}]}$ Luis Crovetto, ${ }^{[\mathrm{a}]}$ Juan A. González-Vera, ${ }^{\left[{ }^{[a]}\right.}$ Eva M. Talavera, ${ }^{[\mathrm{a}]}$ Jose \\ M. Alvarez-Pez, ${ }^{[\mathrm{a}]}$ Noël Boens, ${ }^{*[\mathrm{e}]}$ and María Jose Ruedas-Rama, ${ }^{*[\mathrm{a}]}$ \\ Dedication
}

\begin{abstract}
Three new boron dipyrromethene (BODIPY) dyes substituted with a benzylamino group at the 3-position have been synthesized from halogenated BODIPYs by nucleophilic substitution. The spectroscopic and photophysical properties have been explored in different solvents and have been compared with an analogous 8benzylaminoBODIPY derivative. The position of the benzylamino group has a significant influence on the spectral band positions, fluorescence quantum yields, and fluorescence lifetimes. The 8benzylaminoBODIPY emits in the blue range, whereas the 3substituted shows green fluorescence. Additionally, the extension of the conjugation at the 5-position of 3-benzylaminoBODIPY produces a bathochromic shift of the absorption and emission spectra. The solvent effect on their spectroscopic features has been investigated using the generalized Catalán solvent scales. Quantum-chemical calculations have been performed to assess the effect of the position of the substitution on the spectroscopic properties. Finally, the BODIPY dyes have been employed as probes in fluorescence lifetime imaging of living cells, demonstrating their high potential for bioimaging.
\end{abstract}

\section{Introduction}

[a] C. Ripoll, E. Garcia-Fernandez, A. Orte, L. Crovetto, J. A. GonzálezVera, E. M. Talavera, J. M. Alvarez-Pez, M. J. Ruedas-Rama Department of Physical Chemistry. Faculty of Pharmacy, University of Granada

Campus Cartuja, 18071, Granada, Spain mjruedas@ugr.es

[b] C. Cheng, J. Li, L. Jiao

The Key Laboratory of Functional Molecular Solids, Ministry of Education; School of Chemistry and Materials Science,

Anhui Normal University

Wuhu 241000, China

jiao421@ahnu.edu.cn

[c] H. Do

Department of Chemical and Environmental Engineering, University of Nottingham Ningbo China, 199 Taikang East Road, Ningbo 315100, China

[d] D. Robinson

Department of Chemistry and Forensics, Nottingham Trent University, Clifton Lane, Nottingham, United Kingdom david.robinson@ntu.ac.uk

[e] N. Boens

Department of Chemistry

KU Leuven (Katholieke Universiteit Leuven)

Celestijnenlaan 200f, 3001 Leuven, Belgium Noel.Boens@kuleuven.be

Supporting information for this article is given via a link at the end of the document.
Derivatives of 4,4-difluoro-4-bora-3a,4a-diaza-s-indacene, better known as BODIPY (boron dipyrromethene or boron dipyrrin) dyes ${ }^{[1]}$ have attracted significant attention over the last severa decades, being the focus of considerable research interests because of their extremely desirable photophysical and optoelectronic properties. The outstanding qualities of BODIPY comprise high fluorescence quantum yields $(\Phi)$ and molar absorption coefficients $(\varepsilon)$, narrow absorption and fluorescence emission bands with high peak intensities in the visible region, robustness against light and chemicals, resistance towards selfaggregation in solution, good solubility, neutral net charge, negligible triplet-state formation and fluorescence lifetimes $(\tau)$ in the nanosecond range. Furthermore, another valuable advantage of these fluorophores is their vast potential of functionalization. ${ }^{[2]}$ They are amenable to structural modification so that their spectroscopic and physicochemical properties can be fine-tuned by introducing suitable substituents at the right positions (the pyrrole ring positions, the central 8- or mesoposition, and the boron atom). The facile and efficient functionalization of the BODIPY platform allows for the creation of derivatives with fluorescence emission spanning the whole range of the visible spectrum and extending into the nearinfrared. Consequently, the BODIPY family is growing constantly and has found wide applications in highly diverse fields, from technological, such as organic light-emitting diodes ${ }^{[3]}$ and dyesensitized solar cells, ${ }^{[4]}$ to analytical and in vivo imaging applications. ${ }^{[5]}$ Indeed, the extraordinary photostability and high values of quantum yield and fluorescence lifetime make BODIPY dyes ideally suited for bioimaging and sensing in live cells. ${ }^{[5]}$

Among the fluorescence imaging techniques, fluorescence lifetime imaging microscopy (FLIM) stands out for its multidimensional information, involving both intensity and lifetime data. This allows the development of intracellular sensors free of usual issues that hinder quantitative cellular imaging ${ }^{[6]}$ such as the presence of cellular autofluorescence, and the effect of the local concentration of the probe. Mesosubstituted BODIPY dyes usually exhibit an environmentsensitive fluorescence lifetime because of the rotation of the meso-substituent, and this property has been taken advantage of for the development of intracellular viscosity probes, specifically for FLIM ${ }^{[7]}$ Hence, the search for improved BODIPY cellular probes for FLIM studies remains a matter of great interest.

Boron dipyrromethenes incorporating electron-donating groups at the meso-position display a hypsochromic shift in the absorption and emission spectra, while keeping high fluorescence quantum yields $(\Phi)$ and lifetimes $(\tau){ }^{[8]}$ In this sense, recently, we described the synthesis and photophysical 
properties of the blue-emitting BODIPY 2 (Scheme 1) by attaching a benzylamine at the meso-position of a fully unsubstituted BODIPY. ${ }^{[9]}$ In contrast to classic BODIPY derivatives, this compound exhibited marked solvatochromic behaviour in solvents of different dipolarity. The presence of an amino group directly attached to the 3-position has an additional advantage, as it can establish intramolecular hydrogen bonds with the fluorine atoms, which restricts the torsional motion of the fluorophore, leading to an increase in the fluorescence. ${ }^{[10]}$ Therefore, in the present work, we explore the incorporation of a benzylamino group at position 3 of the BODIPY scaffold (compounds 4 and 8), leading to green-emitting dyes. Likewise, in a previous work, we found that a styryl group placed in position 3 led to stable, highly fluorescent, orange-emitting BODIPY dyes. ${ }^{[8 d]}$ Hence, in this work, we planned to extend the conjugation of the BODIPY core with the styryl group, in a 3benzylamino substituted compound, with the goal of shifting the absorption and emission bands further into the red, given the importance of available near-infrared dyes for in vivo imaging. ${ }^{[11]}$

Thus, in addition to the syntheses of new BODIPY compounds, in the present paper we describe in detail the UVvis photophysical and spectroscopic properties of the benzylamine-substituted BODIPY derivatives. We investigated the effect of the position of the benzylamino group on the absorption and emission spectral maxima [ $\lambda_{\text {abs }}(\max )$ and $\left.\lambda_{\text {em }}(\max )\right]$, the fluorescence quantum yield values $\Phi$, the full width at half height of the absorption (fwhmabs) and emission $\left(\mathrm{fwhm}_{\mathrm{em}}\right)$ bands, the Stokes shift $\left[\Delta \bar{v}=1 / \lambda_{\text {abs }}(\max )-1 / \lambda_{\mathrm{em}}(\max )\right]$, the fluorescence quantum yields $\Phi$, the fluorescence lifetimes $\tau$, and the rate constants of radiative and radiationless deactivation, $k_{\mathrm{f}}$ and $k_{\mathrm{nr}}$. In this study, we pay special attention to general and specific solvent effects over a large range of different solvents and support our experimental results with quantum-chemical calculations. Finally, we demonstrate the potential of these BODIPY dyes for their use as probes for the fluorescence imaging of living cells, and in particular, for FLIM microscopy.

\section{Results and Discussion}

Synthesis of benzylamine-substituted BODIPYs. For the syntheses of the benzylamine-substituted BODIPYs used in this work, halogenated BODIPY precursors were employed. It is well known that 3-halogenated and 8-halogenated BODIPY dyes can undergo addition-elimination nucleophilic aromatic substitution reactions by a wide range of nucleophiles. ${ }^{[8 b, 12]}$

First, 8-chloroBODIPY $\mathbf{1}^{[\mathrm{bb}]}$ reacted smoothly with benzylamine at room temperature, giving meso-benzylaminesubstituted BODIPY 2 in $73 \%$ yield (Scheme 1).

3-Benzylamine-substituted BODIPY 4 was synthesized in $68 \%$ yield by reacting 3 -chloroBODIPY $3{ }^{[13]}$ with 2 equivalents of benzylamine in 1,2-dichloroethane under reflux conditions for 0.5 h. 5-styrylated 3-benzylamine-substituted BODIPY 6 was obtained in $63 \%$ yield from BODIPY $5^{[14]}$ using excess benzylamine in 1,2-dichloroethane under reflux conditions for 2 h (Scheme 1).

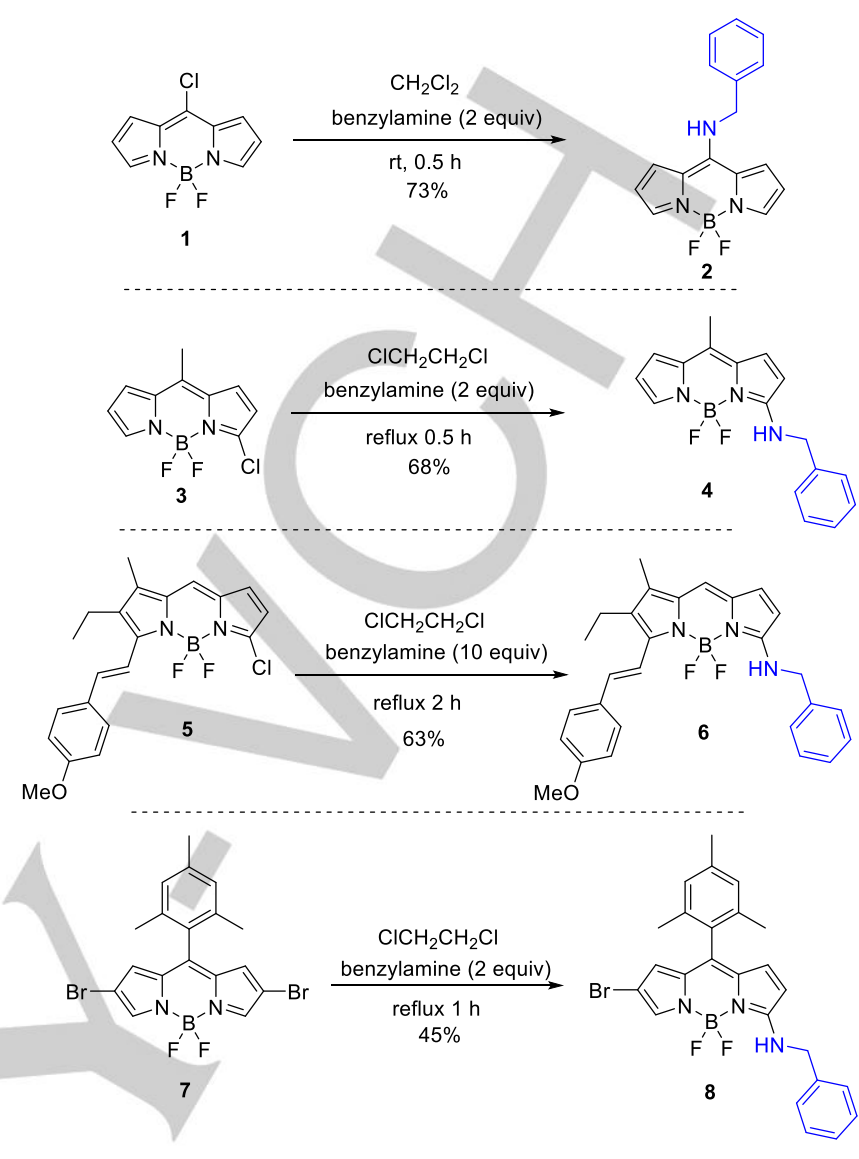

Scheme 1. Synthesis of BODIPY dyes 2, 4, 6 and 8.

Finally, the 3-benzylamine-substituted BODIPY 8 was synthesized from brominated BODIPY $7^{[14]}$ and benzylamine using the copper-catalysed amination reported by Ma et al. ${ }^{[15]}$ However, compound $\mathbf{8}$ was unexpectedly obtained, as the reaction was planned to yield a 2-benzylamine-substituted BODIPY (see Scheme S1 in the Supporting Information, SI, for more details). Instead, the benzylamino group was attached to position 3, with replacement of the $2-\mathrm{Br}$ atom by a hydrogen atom. The structure of 8 was confirmed by ${ }^{1} \mathrm{H}$ and ${ }^{13} \mathrm{C}$ NMR, HRMS (High Resolution Mass Spectrometry) and X-ray structure analysis (Figure 1, and Table S1). The unexpected substitution at the 3-position may be the result of the fact that the electron withdrawing $\mathrm{Br}$ atoms at the 2,6-positions further increase the reactivity of the 3,5-positions of BODIPY towards benzylamine. Moreover, the carbons at the 2,6-positions have the highest electron densities, making nucleophilic substitution at these positions very difficult. In fact, substitution with $\mathrm{N}$-nucleophiles at the 2,6-positions of BODIPY dyes has not been reported. Indeed, BODIPY 8 was obtained directly in $45 \%$ yield from compound 7 using only 2 equivalents of benzylamine under reflux conditions for $1 \mathrm{~h}$ in 1,2-dichloroethane without any catalyst.

The structures of BODIPY 2 (CCDC 1588596) and 8 (CCDC 1588597) (Figure 1) were analysed by single-crystal $X$-ray diffraction (Table S1). The $\mathrm{C}_{9} \mathrm{~N}_{2}$ dipyrrin cores in both dyes are planar, with maximum deviations of only $0.07 \AA$ for 2 and $0.06 \AA$ 
for 8 . The angles between the two pyrrole rings are $4.84^{\circ}$ for 2 and $5.59^{\circ}$ for 8 (Table S2). The data indicate that there is no steric hindrance of the benzylamino substituents to cause structural distortion of the dipyrrin cores. For both BODIPYs 2 and $\mathbf{8}$, the substituents at the $\mathrm{N}$ atoms are coplanar, and the C1-N3 bond distances are 1.33 and $1.34 \AA$, respectively, which are shorter than a typical single $\mathrm{C}-\mathrm{N}$ bond. In contrast, the C2-N3 bond distances are $1.46 \AA$ for 2 and $1.47 \AA$ for 8 (Table S2). These data indicate that the lone pair of electrons on the $N$ atom is conjugated with the planar dipyrrin core. Similar to that of the parent BODIPY, ${ }^{[16]}$ the boron centre of compound 2 adopts a slightly distorted tetrahedral geometry with $\mathrm{N}-\mathrm{B}-\mathrm{N}, \mathrm{F}-$ $\mathrm{B}-\mathrm{F}$, and $\mathrm{N}-\mathrm{B}-\mathrm{F}$ bond angles of $105.9^{\circ}, 108.7^{\circ}$, and $110.6^{\circ}$ (averaged values of four $\mathrm{N}-\mathrm{B}-\mathrm{F}$ bond angles), respectively. Similarly, these values are $106.3^{\circ}, 106.1^{\circ}$, and $111.1^{\circ}$ for BODIPY 8. Further details of the synthesis reactions and their structural characterization can be found in the SI.

A

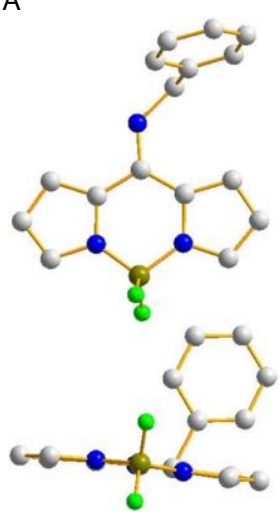

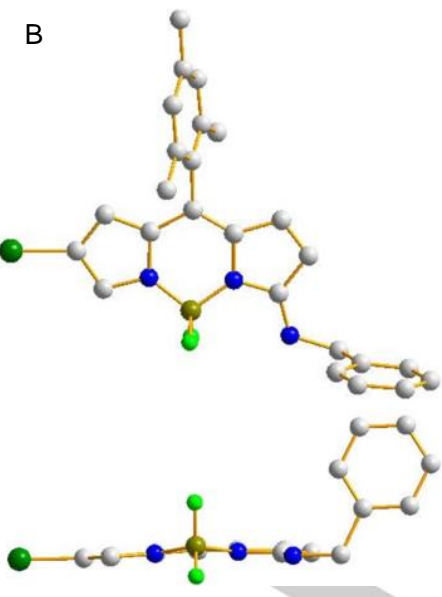

Figure 1. Top and front views of X-ray crystal structure of $2(A)$ and $8(B)$ : C, light grey; $\mathrm{N}$, blue; $\mathrm{B}$, dark yellow; $\mathrm{F}$, bright green; $\mathrm{Br}$, green.

Spectroscopic and photophysical properties. The newly synthesized BODIPY derivatives are strongly coloured solids that form intensely coloured solutions with bright fluorescence upon irradiation. BODIPYs 2, 4 and $\mathbf{6}$ were dissolved in 19-20 different solvents, and their UV-vis absorption and fluorescence emission spectra were collected and compared (Tables 1, S3 and S4). Although the full characterization of the 8-benzylaminosubstituted 2 has been reported previously by us, ${ }^{[9]}$ we include it in this work for discussion purposes. Dye $\mathbf{4}$ exhibits typical BODIPY-like absorption spectra in all solvents tested, ${ }^{[1 \mathrm{~b}, 1 \mathrm{c}]}$ with a narrow band because of the $S_{1} \leftarrow S_{0}$ transition (Figure 2). As reported previously, compound $\mathbf{2}$ with the benzylamino substituent at the 8-position absorbs and emits in the blue range of the visible spectrum and has larger Stokes shifts than common BODIPYs. ${ }^{[9]}$ When the benzylamine group is at the 8position, BODIPY dye 2 exhibits absorption maxima from 400 to $422 \mathrm{~nm}$, but when it is at the 3-position (compound 4) the absorption is shifted bathochromically $(\sim 60-80 \mathrm{~nm})$ to $462-504$ $\mathrm{nm}$, and exhibits a small bathochromic shift with increasing solvent polarizability. In both cases, the longest absorption wavelength is found in cyclohexane, and the lowest $\lambda_{\text {abs }}(\max )$ value is observed in acetonitrile (Tables 1, S3 and S4). Figure 2 shows representative examples of the visible electronic absorption and fluorescence emission spectra of $\mathbf{4}$ in a selection of solvents. In more polar solvents, the broadening of the individual vibronic bands because of solvent interactions blurs out the vibrational progression observed in cyclohexane. Likewise, another difference between 8- and 3-substitution arises in the analysis of the spectral widths. Whereas compound

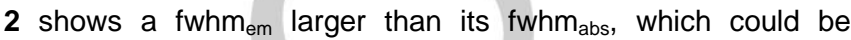
attributed to a decreased rigidity in the excited state, compound 4 displays the contrary trend (Tables 1, S3 and S4).

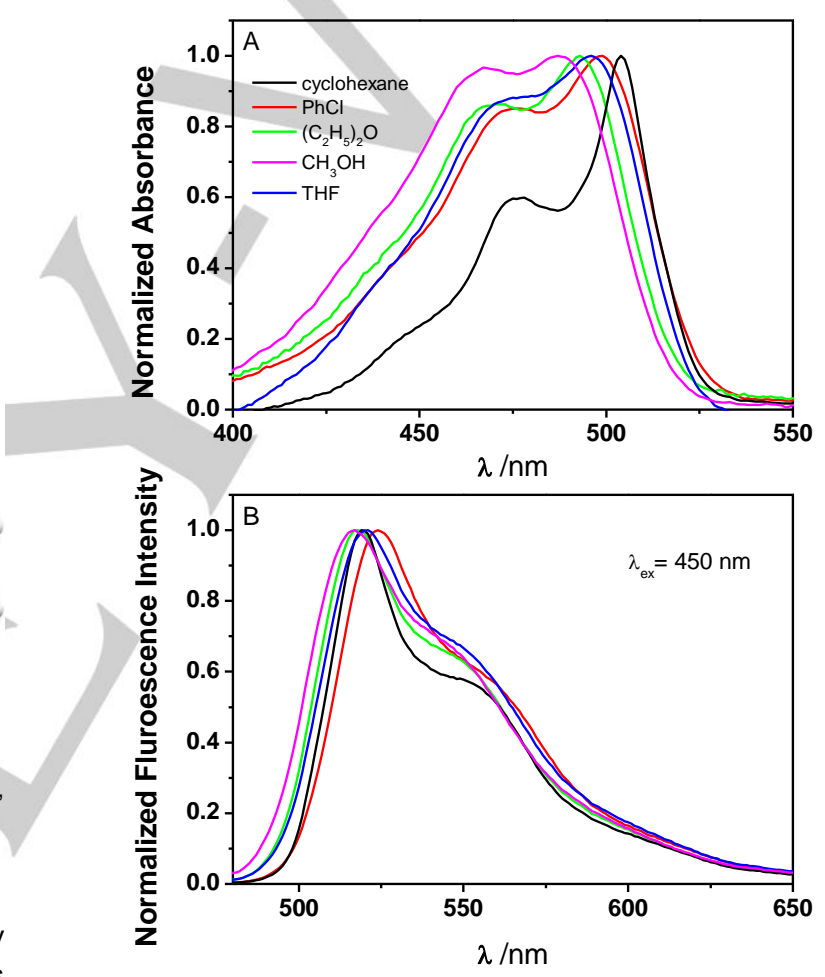

Figure 2. Normalized main $S_{1} \leftarrow S_{0}$ visible absorption band $(A)$ and normalized fluorescence emission spectra $(B)$ of $\mathbf{4}$ in the solvents indicated.

The spectroscopic features of compound $\mathbf{4}$ are rather similar to an analogous 3-phenylaminoBODIPY derivative (compound 10 in Figure 3). The unsymmetrically substituted dye $\mathbf{1 0}$ with a phenylamine moiety at 3-position showed absorption spectra dependent on solvent dipolariry, exhibiting a total shift of ca. 30 $\mathrm{nm}$ in the absorption maximum (from $498 \mathrm{~nm}$ in methanol to 529 $\mathrm{nm}$ in cyclohexane) ${ }^{[10 \mathrm{~b}]}$. In both cases, the fwhmabs is in the $2500-3500 \mathrm{~cm}^{-1}$ range, which is significantly higher than those of other BODIPY derivatives with weak electron donating or electron withdrawing groups at the 3 - or 5-position. ${ }^{[17]}$ These broad absorption spectra of $\mathbf{4}$ and the 3-phenylamino-substituted counterpart $\mathbf{1 0}$ are because both dyes have a relatively strong 
bond alternation in the ground state, which changes upon excitation. The emission spectra of both compounds also show similar shapes. In addition to the 0-0 main transition, there is the 0-1 transition, visible as a shoulder peak (Figure 2). Another common feature of both dyes is that the emission maxima are hardly affected by solvent dipolariry. The difference in the emission maximum wavelength of the dyes dissolved in methanol or toluene is only $9 \mathrm{~nm}$ for compound $10^{[10 \mathrm{~b}]}$ and $7 \mathrm{~nm}$ for compound 4 (Table S4).

The position of the benzylamino group also affects the $\Phi$ values of compounds $\mathbf{2}$ and $\mathbf{4}$ in different solvents. The $\Phi$ of the 8-substituted compound $\mathbf{2}$ is solvent dependent, increasing from 0.08 in methanol to 0.75 in toluene. ${ }^{[9]}$ The 3-benzylamino compound 4 exhibits larger $\Phi$ values than 2 , varying from 0.78 in methanol to 0.94 in cyclohexane and cyclohexanone; however, for this dye there is a less noticeable dependence on the dipolariry of the solvent (Tables 1 and S4). On the other hand, both benzylaminoBODIPY dyes, 2 and $\mathbf{4}$, show an enhancement in the $\Phi$ values compared with their corresponding phenylamino derivatives. In a previous work, it was observed that when the phenylamino group is at the 8-position the BODIPY dye is practically non-fluorescent, ${ }^{[9,}{ }^{18]}$ which was attributed to an acceleration of the internal conversion by coupling with an electron transfer state. However, when the 8-substituent is benzylamine (compound 2), the compound is fluorescent, although with large variations in the $\Phi$ values dependent on solvent dipolarity. ${ }^{[9]}$ BODIPY 2 exhibits a dipolariry dependence similar to other BODIPY derivatives with a $p$-anilino substituent in the meso-position. ${ }^{[19]}$ The comparison of phenylamino and benzylamino substituents at the 3-position also shows a similar trend. Whereas the 3-phenylamino derivative is only moderately fluorescent in cyclohexane $(\Phi=0.28)$, chloroform $(\Phi=0.26)$ and toluene $(\Phi=0.35)$, it is non-fluorescent in polar solvents; $;^{[10 b]}$ the quantum yield of the 3-benzylaminoBODIPY $\mathbf{4}$ increases drastically up to an average value of 0.85 in all solvents tested (Table S4), and as discussed before, shows very little dipolariry dependence.

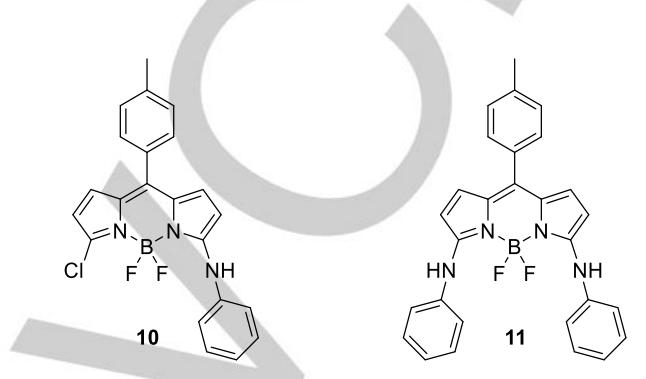

Figure 3. Chemical structures of BODIPY dyes discussed in this paper: 3 phenylaminoBODIPY derivative 10 and 3,5-diphenylaminoBODIPY derivative 11.

Table 1. Spectroscopic and photophysical data of $\mathbf{4}$ and $\mathbf{6}$ in selected solvents.

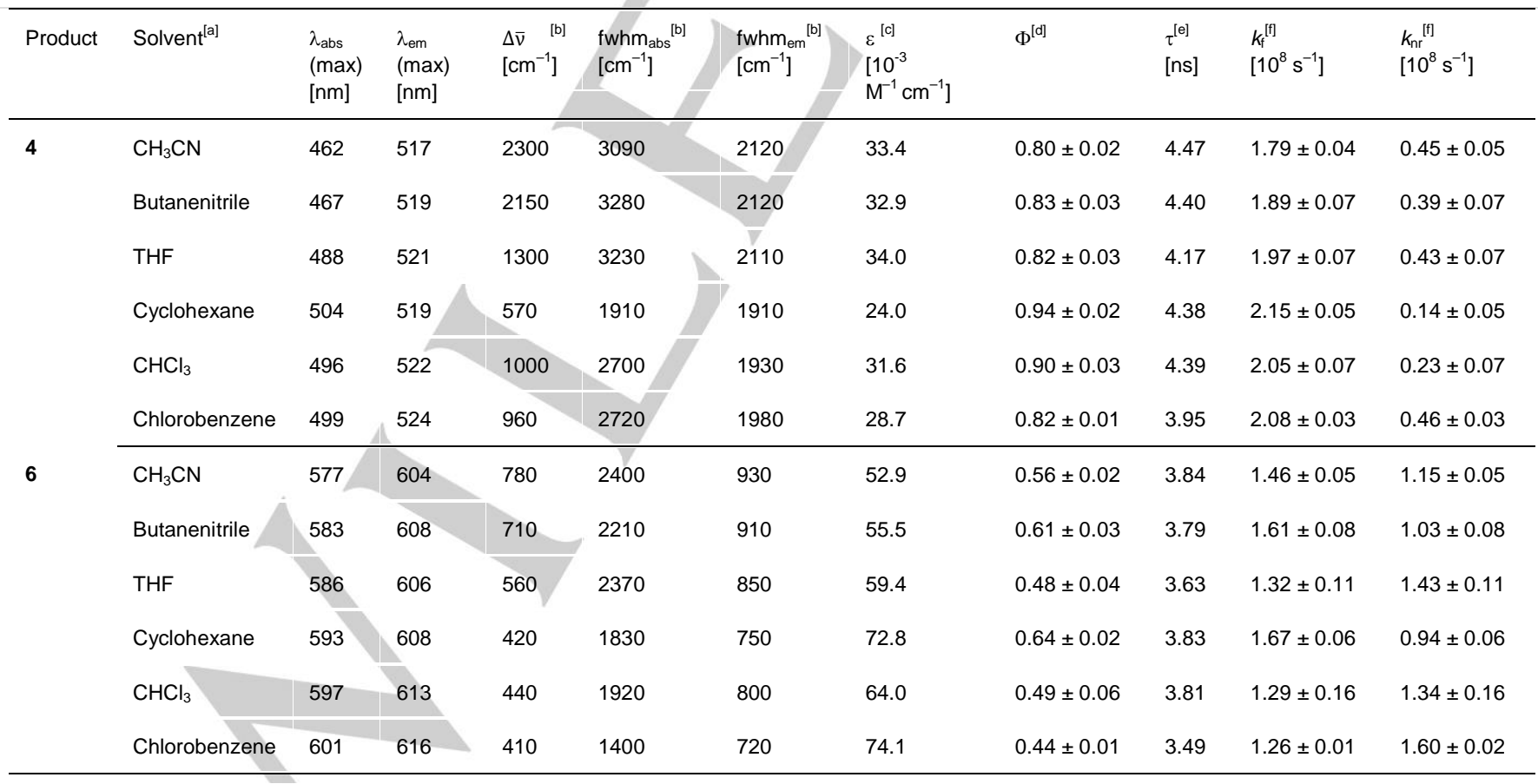

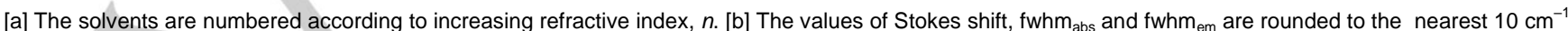
[c] The values of absorption coefficients, $\varepsilon$, are rounded to the nearest $100 \mathrm{M}^{-1} \mathrm{~cm}^{-1}$. [d] Fluorescence quantum yield \pm one standard uncertainty. $\Phi$ determined using fluorescein in $0.1 \mathrm{~N} \mathrm{NaOH}\left(\Phi_{\mathrm{r}}=0.91\right)$ as a reference for 4, and rhodamine 101 in ethanol $\left(\Phi_{\mathrm{r}}=0.96\right)$ as a reference for $\mathbf{6}$. [e] Fluorescence lifetimes obtained with $\lambda_{\mathrm{ex}}=485 \mathrm{~nm}$, and $\lambda_{\mathrm{em}}=520,530,540,550 \mathrm{~nm}$ for 4 , and with $\lambda_{\mathrm{ex}}=530 \mathrm{~nm}$ and $\lambda_{\mathrm{em}}=605,610,615,620 \mathrm{~nm}$ for 6 . The standard errors were 
obtained from the diagonal elements of the covariance matrix available from the global analysis fit of decay traces recorded at four emission wavelengths and were between 7 and 10 ps for 4 and between 11 and 41 ps for 6 . [f] The propagated errors were calculated using the uncertainty (standard deviation) of $\Phi$ and the standard error of $\tau$.
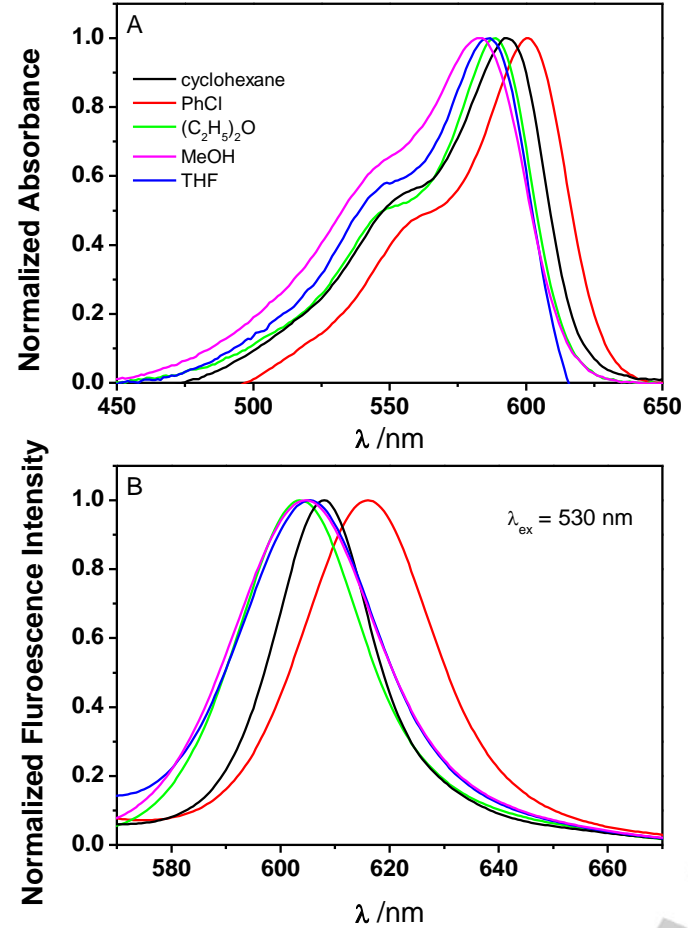

Figure 4. Normalized main $S_{1} \leftarrow S_{0}$ visible absorption bands $(A)$ and normalized fluorescence emission spectra $(B)$ of 6 in the solvents indicated.

The incorporation of a 4-methoxyphenylethenyl substituent at the 5-position considerably changes the spectroscopic features, as evidenced in compound 6 . The normalized absorption and fluorescence emission spectra of BODIPY 6 dissolved in five different solvents are shown in Figure 4. As has been reported, by increasing the conjugation at the 3,5-positions, the absorption and emission spectra can be shifted to longer wavelengths. ${ }^{[17,20]}$ The maximum absorption wavelength of compound 6 (3,5-disubstituted) is shifted to the red in comparison with the 3-monosubstituted dye 4. The extended conjugation provided by the styryl functional group causes an extra bathochromic shift. In all the solvents tested, the main absorption band, attributed to the $0-0$ vibrational band of a strong $S_{1} \leftarrow S_{0}$ transition, is situated near $590 \mathrm{~nm}$, with a shoulder at shorter wavelengths and is assigned to the 0-1 vibrational band of the same transition. Moreover, the $\lambda_{\text {abs }}$ (max) is also red-shifted with increasing polarizability of the solvent, from $577 \mathrm{~nm}$ in acetonitrile to $601 \mathrm{~nm}$ in chlorobenzene (Tables 1 and S5). The fwhm ${ }_{a b s}$ and, especially, fwhm $\mathrm{em}_{\mathrm{e}}$ of $\mathbf{6}$ are always significantly narrower than the corresponding ones for compound 4, indicating that for the disubstituted BODIPY there is substantially less bond alternation upon excitation. ${ }^{[10 b]}$ Compound 6 displays smaller Stokes shifts, narrower emission bands, and more red-shifted $\lambda_{\mathrm{em}}(\max )$ values $(\sim 90 \mathrm{~nm})$ than those from BODIPY 4 . The $\lambda_{\mathrm{em}}(\max )$ values of 6 also show dependence with the polarizability of the solvent, from $604 \mathrm{~nm}$ in acetonitrile to $616 \mathrm{~nm}$ in chlorobenzene (Tables 1 and S5). However, the $\Phi$ values of $\mathbf{6}$ are lower than those of compound $\mathbf{4}$, with 0.54 being the average for all the solvents tested. As is evident from Tables $1, \mathrm{~S} 4$ and $\mathrm{S} 5$, the $\Phi$ values are also nearly independent of solvent polarizability.

The spectral features of compound 6 can also be compared with those of an analogue 3,5-diphenylamino-substituted BODIPY dye ${ }^{[21]}$ (compound 11 in Figure 3), finding a large number of similarities. The shapes of the absorption and emission bands are very alike, and both dyes show large bathochromic shifts in their $\lambda_{\text {abs }}(\max )$ and $\lambda_{\text {em }}(\max )$ values. Both absorption and emission maxima are blue-shifted with decreasing polarizability of the solvent for compound 6 (Tables 1 and S5) and the 3,5-diphenylaminoBODIPY 11. ${ }^{[1]}$ In addition, both 3,5-disubstituted dyes display narrower fwhmabs and fwhm $_{\mathrm{em}}$ values than the corresponding 3-monosubstituted counterparts. This indicates that the latter ones have a higher bond alternation in the ground state, which changes upon excitation, but for the 3,5-disubstituted dyes, the major delocalized forms have a similar contribution in both the ground and the excited states.

We also obtained information on the excited-state kinetics through fluorescence decay measurements of $\mathbf{4}$ and $\mathbf{6}$ in the 20 and 19 solvents tested, respectively. We obtained the fluorescence lifetimes $\tau$ and calculated the rate constants for radiative $\left(k_{\mathrm{f}}=\Phi / \tau\right)$ and radiationless $\left[k_{\mathrm{nr}}=(1-\Phi) / \tau\right]$ deactivation. Unlike compound 2, whose fluorescence lifetime is clearly solvent dependent and increases from $0.57 \mathrm{~ns}$ in methanol to $4.90 \mathrm{~ns}$ in cyclohexane ${ }^{[9]}$ the solvent exhibits a negligible effect on the fluorescence lifetimes $\tau$ of $\mathbf{4}$ and $\mathbf{6}$ (Tables 1, S4 and S5). For compound 4 , the $\tau$ values vary between $3.95 \mathrm{~ns}$ in chlorobenzene and 4.63 ns in diethyl ether, and for 6 , between $3.49 \mathrm{~ns}$ in chlorobenzene and $4.05 \mathrm{~ns}$ in 1 -octanol. Illustrative examples of fluorescence decay traces of $\mathbf{4}$ and $\mathbf{6}$ in different solvents are shown in Figures S2 and S3 (SI). The radiative deactivation rate constant $\left(k_{f}\right)$ is practically solvent independent for each of the three dyes investigated: $(1.5 \pm 0.1) \times 10^{8} \mathrm{~s}^{-1}$ for 2 , $(2.0 \pm 0.1) \times 10^{8} \mathrm{~s}^{-1}$ for 4 , and $(1.4 \pm 0.2) \times 10^{8} \mathrm{~s}^{-1}$ for 6 (Tables $1, \mathrm{~S} 3, \mathrm{~S} 4$ and S5). The non-radiative rate constant $\left(k_{\mathrm{nr}}\right)$ is also nearly independent of the solvent for BODIPYs 4 and 6 [ $0.3 \pm$ $0.1) \times 10^{8} \mathrm{~s}^{-1}$ for 4 and $(1.2 \pm 0.2) \times 10^{8} \mathrm{~s}^{-1}$ for 6$]$, whereas for BODIPY 2 the $k_{\mathrm{nr}}$ values decrease with the solvent polarizability (Tables S3). ${ }^{[9]}$

Finally, the UV-vis absorption and fluorescence emission spectra of the 3-benzylamino-substituted BODIPY 8 were collected in a few select solvents and compared with those of BODIPY 4. As can be observed in Figure S4, the shapes of the absorption and emission spectra of $\mathbf{8}$ and $\mathbf{4}$ (Figure 2) are alike, and the $\lambda_{\text {abs }}(\max )$ and $\lambda_{\text {em }}(\max )$ are also similar, with an insignificant bathochromic shift of 8 compared with that of 4 (Table S4 and Table S6). In addition, in both cases, the values 
of the spectroscopic $\lambda_{\text {abs }}(\max )$ and $\lambda_{\mathrm{em}}(\max )$ increase with increasing solvent polarizability. These results indicate that the position of the benzylamino group is the main parameter responsible of the spectral characteristics of the BODIPY dyes. However, the $\Phi$ values of $\mathbf{8}$ are slightly lower than those of compound 4 , presumably because of the presence of a bromine atom in 8.

\section{Solvatochromism}

It is instructive to discover the origin of the solvent-dependent spectroscopic changes, using the analysis tool provided by Catalán. ${ }^{[22]}$ This generalized treatment of the solvent effect is based on four empirical, complementary, mutually independent solvent scales, i.e., the dipolarity, polarizability, acidity and basicity of the medium. The polarizability and dipolarity of a specific solvent are characterized by the indices SP and SdP, respectively, whereas solvent acidity and basicity are defined by the scales SA and SB, respectively. The $\{\mathrm{SA}, \mathrm{SB}, \mathrm{SP}, \mathrm{SdP}\}$ parameters that characterize respectively the solvent acidity, basicity, polarizability and dipolarity for a large number of solvents can be found in the work of Catalán. ${ }^{[22]}$ Mathematically, the solvent effect on the physicochemical observable $y$ can be expressed by the multilinear expression 1 :

$$
y=y_{0}+a_{\mathrm{SA}} \mathrm{SA}+b_{\mathrm{SB}} \mathrm{SB}+c_{\mathrm{SP}} \mathrm{SP}+d_{\mathrm{SdP}} \mathrm{SdP}
$$

where $y_{0}$ denotes the physicochemical property of interest in the gas phase; asA, $b_{\mathrm{SB}}, c_{\mathrm{SP}}$ and $d_{\mathrm{SdP}}$ are (adjustable) regression coefficients that reflect the dependency of the property $y$ on the various solvent-solute interaction mechanisms; and $\{\mathrm{SA}, \mathrm{SB}, \mathrm{SP}$, $\mathrm{SdP}\}$ are mutually independent solvent parameters (or indices).

The spectroscopic observable $y$ values analysed in this paper are the absorption maxima, $\bar{v}_{\text {abs }}\left[=1 / \lambda_{\text {abs }}(\max )\right]$, and the fluorescence emission maxima, $\bar{v}$ em $\left[=1 / \lambda_{\text {em }}(\max )\right]$, both expressed in $\mathrm{cm}^{-1}$. We focused on those parameters that exhibited clear solvent dependence, whereas other properties, such as $k_{f}$ and $k_{n r}$, did not show such a dependence, and therefore, could not be described by eqn. 1 . The results of the fits of $y=\bar{v}_{\text {abs }}$ and $y=\bar{v}_{\text {em }}$ according to eqn. 1 for BODIPYs 4 and 6 are compiled in Table S7 (SI). Use of the Catalán solvent scales produced excellent fits of $y=\bar{v}_{\text {abs }}$ for the solvents listed in Tables S4 and S5, using the correlation coefficient $r$ as a goodness-of-fit criterion ( $r=0.909$ for 4 , and $r=0.937$ for 6 ). Comparable high-quality fits were obtained for the multilinear analysis of $y=\bar{v}_{\text {em }}$ according to eqn. 1 ( $r=0.960$ for $\mathbf{4}$ and 6).

The unique, outstanding feature and the distinct advantage of the generalized treatment of the solvent effect over all the other approaches is that it allows one to separate the relative contributions of dipolarity, polarizability, acidity and basicity of the medium. Therefore, we utilized the Catalán methodology to resolve which solvent properties are primarily responsible for the observed solvatochromic shifts of $\bar{v}_{\text {abs }}$ and $\bar{v}_{\text {em }}$. The relative importance of each of the Catalán solvent scales was studied by omitting in turn one, two or three solvent scales from the regression analysis (eqn. 1).

Now, we discuss in detail the solvatochromic analyses of $\bar{v}_{\text {abs }}$ and $\bar{v}_{\text {em }}$ of BODIPY 6 . The high-quality fit $(r=0.937)$ of $y=\bar{v}_{\text {abs }}$ of 6 , according to eqn. 1 with $\{\mathrm{SA}, \mathrm{SB}, \mathrm{SP}, \mathrm{SdP}\}$ as independent $x$ variables, yielded large negative $C_{S P}$ and positive $d_{S d P}$ estimates with high precision (i.e., comparatively small standard error of $\sim 17 \%$ ) compared with the small $a_{\mathrm{SA}}$ and $b_{\mathrm{SB}}$ estimates with relatively high standard errors (up to $140 \%$ for $b_{S B}$, Table S7). This is indicative that the change of $\bar{v}_{\text {abs }}$ predominantly reflects a change in the polarizability and dipolarity of the environment of the dye. If either SP or SdP was left out as an independent $x$ variable in the analyses of $\bar{v}_{\text {abs }}$ of $\mathbf{6}$, then rather low correlation coefficient values were found $(0.744$ for $\{S A, S B, S d P\}$ and 0.748 for $\{S A, S B, S P\}$ ), implying the importance of these solvent parameters. In contrast, the two analyses of $y=\bar{v}_{\text {abs }}$ according to eqn. 1 with both $\mathrm{SP}$ and SdP present gave excellent fits (Table S7). Of the six analyses with two solvent scales as independent $x$ variables, five gave $r \leq 0.747$ values; only the analysis including both $\{\mathrm{SP}, \mathrm{SdP}\}$ yielded a higher $r$ value $(0.913)$, corroborating the key role of solvent polarizability and dipolarity in influencing the position of the absorption maxima. That solvent acidity and basicity have a negligible effect on $\bar{v}_{\text {abs }}$, as evident from the unacceptable fit $(r=0.367)$ for the analysis with $\{\mathrm{SA}, \mathrm{SB}\}$ as independent $x$ variables. None of the simple linear regression analyses with a single independent $x$ variable gave an acceptable fit, proving that the solventdependent shifts of $\bar{v}_{\text {abs }}$ are not governed by a single solvent parameter.

The Catalán $\{\mathrm{SA}, \mathrm{SB}, \mathrm{SP}, \mathrm{SdP}\}$ solvent scales also excellently described the solvatochromic shifts of $\bar{v}_{\text {em }}$ of dye $6(r$ $=0.960$, Table S7). However, the situation differed markedly from the analysis of $\bar{v}_{\text {abs }}$, because the very large negative $c_{S P}$ estimate with comparatively small standard error (<10\%) dominated the substantially smaller $a_{\mathrm{SA}}, b_{\mathrm{SB}}$ and positive $d_{\mathrm{SdP}}$ estimates with relatively high standard errors (up to $85 \%$ for $d_{S d P}$, Table S7). This clearly shows that the solvent-dependent shifts of $\bar{v}$ em reflect primarily a change in polarizability of the environment surrounding the dye. To corroborate this, we performed - as for $\bar{v}_{\text {abs }}$ - some additional regression analyses of $y=\bar{v}_{\text {em }}$ according to eqn. 1 , in which systematically one, two or three solvent scales were omitted. These analogous analyses of $\bar{v}_{\text {em }}$ of compound $\mathbf{6}$ substantiated that solvent polarizability alone determines the position of $\bar{v}_{\text {em }}$. For instance, the analyses of $\bar{v}_{\text {em }}$ in which one solvent scale was left out gave excellent fits on the condition that SP was present as an independent $x$ variable in eqn. 1 (Table S7). In contrast, the analysis of $y=\bar{v}_{\mathrm{em}}$ according to eqn. 1 , in which solvent polarizability was disregarded, produced a low $r$-value (Table S7). The analyses of $y=\bar{v}_{\text {em }}$ according to eqn. 1 with two solvent parameters produced acceptable fits provided that SP was present as an independent $x$ variable (Table S7). Even the simple linear regression of $y=$ $\bar{v}_{\mathrm{em}} v s \mathrm{SP}$ as the independent $x$ variable still gave a good fit $(r=$ 0.915). The large negative $c_{S P}$ estimate is in agreement with the observed bathochromic shifts of $\lambda_{\text {em }}(\max )$ (i.e., smaller $\bar{v}_{\mathrm{em}}$ values) with increasing solvent polarizability, SP (or refractive index $n$ ), as observed in tables S4 and S5 in going from methanol $(n=1.3288, \mathrm{SP}=0.608)$ to chlorobenzene $(n=1.5241$, $\mathrm{SP}=0.833)$. For 6 , there is also a good correlation between $y=$ $\bar{v}_{\text {em }}$ and $n^{2}(r=0.905)$ or the Bayliss function $\left[f\left(n^{2}\right)=\left(n^{2}-1\right) /\left(2 n^{2}\right.\right.$ $+1)$ ] $(r=0.899)$. This is additional evidence that van der Waals and excitonic interactions with a polarizable solvent are primarily 
responsible for the experimentally observed solvent-dependent shifts of $\bar{v}_{\text {em. }}{ }^{[23]}$

Similar analyses of $y=\bar{v}_{\text {abs }}$ and $y=\bar{v}_{\text {em }}$ of 4 (Table S7), according to eqn. 1 , showed that solvent polarizability and dipolarity are also the major causes for the solvatochromic shifts of the absorption maxima $\left(\bar{v}_{\text {abs }}\right)$, whereas solvent polarizability solely determines the fluorescence emission maxima $\left(\bar{v}_{\text {em }}\right)$. For the fluorescence emission maxima of dye 4, the goodness-of-fit remained practically unvaried for analyses according to eqn. 1 with four, three, two or one solvent scales, as long as solvent polarizability (SP) was included in the analysis ( $r$ changes from 0.960 for $\{\mathrm{SA}, \mathrm{SB}, \mathrm{SP}, \mathrm{SdP}\}$ to 0.953 for $x=\mathrm{SP}$ ), corroborating the importance of this solvent parameter for the position of $\bar{v}_{\mathrm{em}}$. Compared with BODIPY 6, a somewhat better correlation was found between $y=\bar{v}_{\text {em }}$ of dye $\mathbf{4}$ and the independent $x$ variables $n^{2}(r=0.921)$ or $f\left(n^{2}\right)(r=0.925)$.

The Catalán analyses of the 3-benzylaminoBODIPYs 4 and 6 corroborate that solvent acidity and basicity have negligible effects on the positions of $\bar{v}_{\text {abs }}$ and $\bar{v}_{\text {em }}$; polarizability is the dominant parameter influencing the solvent-dependent shifts, with solvent dipolarity being a minor contributor. The negative $c_{S P}$ estimates account for the bathochromic shift of the spectroscopic observables, $\lambda_{\text {abs }}(\max )$ and $\lambda_{\mathrm{em}}$ (max), with increasing solvent polarizability. This has also been found for BODIPY dyes $\mathbf{1 0}$ and $\mathbf{1 1}$ with phenylamino substituents at the 3(,5)-position(s). ${ }^{[21]}$

The solvent dependency of $\bar{v}$ abs and $\bar{v}$ em for 8benzylaminoBODIPY 2 differs quite clearly from those of 3 benzylaminoBODIPYs 4 and $\mathbf{6}$ and most BODIPYs investigated earlier, where solvent polarizability is the main cause for the solvent dependence of the absorption and fluorescence emission maxima. ${ }^{[9]}$ In a previous paper, we discussed in detail the solvatochromism of $\bar{v}_{\text {abs }}$ and $\bar{v}_{\text {em }}$ of 2 , and we refer to this reference for an extensive discussion thereof. However, its accompanying Supporting Information included only limited data on the results of the various multilinear regression analyses. Therefore, we re-analysed the $\bar{v}_{\text {abs }}$ and $\bar{v}_{\text {em }}$ data of $\mathbf{2}$ to present extended data on the results in Table S8.

The key role of solvent dipolarity in determining the position of the absorption maxima of $\mathbf{2}$ was evident from the regression analyses, whereby in turn one, two or three independent $x$ variables were omitted from the regression (eqn. 1). As long as $\mathrm{SdP}$ was included in the analysis, good/satisfactory fits (high $r$ values) were obtained, whereas poor/unacceptable fits were found when SdP was dropped from eqn. 1 (Table S8). The regression analyses of $\bar{v}_{e m}$ of 2 according to eqn. 1, whereby systematically one independent $x$ variable was cut from the equation, atributed to identified solvent basicity (and, to a lesser degree, solvent dipolarity) as critical factors influencing the fluorescence emission maxima (Table S8). The regression analyses of $\bar{v}_{\text {em }}$ with two independent $x$ variables produced the best fit when both SB and SdP were present $(r=0.924)$. When $\mathrm{SB}$ was included in the regression analyses of $\bar{v}_{\mathrm{em}}$ with two independent $x$ variables, fits with $r$-values $\geq 0.829$ were obtained (Table S8). Conversely, when SdP was included in the regression analyses of $\bar{v}_{\mathrm{em}}$ with two independent $x$ variables, fits with somewhat lower $r$-values were obtained $(r=0.731$ for $\{\mathrm{SA}$,
$\mathrm{SdP}\}$ and $r=0.775$ for $\{\mathrm{SP}, \mathrm{SdP}\}$, Table S8), highlighting the minor role of solvent dipolarity compared with the major influence of solvent basicity in affecting the position of the fluorescence emission maxima. This was also evident from the simple regression analyses of $\bar{v}_{\mathrm{em}} v s \mathrm{SB}(r=0.822)$ and $v s \mathrm{SdP}$ $(r=0.634)$ (Table S8).

\section{Quantum-chemical calculations}

Computational descriptions of BODIPY substituents have proven very useful in understanding the photophysical properties of the dyes. In previous studies, we demonstrated the non-planar nature of the BODIPY core in the $S_{1}$ excited state. ${ }^{[24]}$ We also demonstrated that bulky substituents, such as tert-butyl, at the 8-position cause very distorted, non-planar $\mathrm{S}_{1}$ geometries, which lead to ultrafast relaxation via a conical intersection. ${ }^{[8 d, 25]}$ Likewise, previously, the change in the charge density upon excitation from the $\mathrm{S}_{0}$ state to the $\mathrm{S}_{1}$ state has been linked to the hypsochromic shift observed with increasing dipolariry of the solvent. ${ }^{[10 b]}$
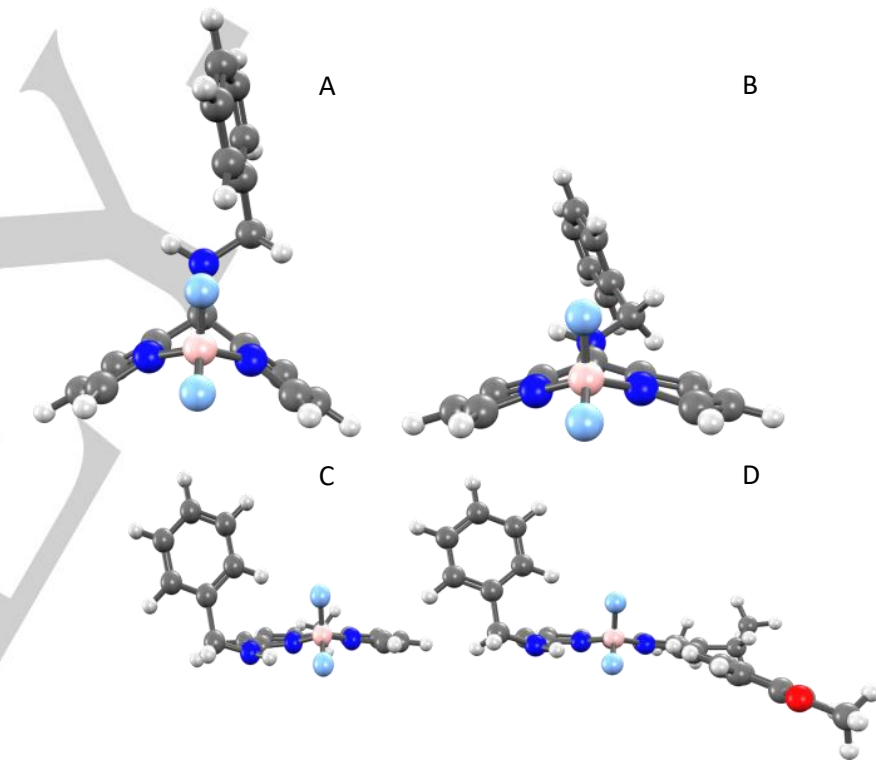

Figure 5. Calculated $S_{1}$ geometries for 2 (gas phase, A; solvated in toluene, B), 4 (gas phase, C) and 6 (gas phase, D).

Given in Figure 5 are the optimised $S_{1}$ geometries for 2 in the gas phase $(A)$ and solvated in toluene $(B)$; the gas-phase excited-state geometries of 4 (C) and 6 (D) are also given. As we have observed previously, bulky groups at the 8-position cause distorted geometries in the $\mathrm{S}_{1}$ excited state, with the BODIPY core becoming very non-planar. This is observed in the excited-state geometry of $\mathbf{2}$, especially in the gas phase. The excited-state geometry of $\mathbf{2}$ when solvated in toluene exhibits significantly less distortion of the BODIPY core. The solvent dependence of this distortion hints at the competition between fluorescence and radiationless decay via a conical intersection, as observed for 2 in toluene (emission observed) and methanol (emission not observed). This behaviour is not seen for 
compounds $\mathbf{4}$ and $\mathbf{6}$, where the BODIPY core remains essentially planar, which correlates with larger emission efficiencies, $\Phi$.

To understand the change in the electron density upon excitation, the natural transition orbitals can be calculated. ${ }^{[26]}$ The natural orbitals are so termed as their eigenvalues correspond to the orbital occupation numbers. These give localized regions of the molecule for the "hole" and "particle" densities of the exciton, which essentially correspond to the pair of orbitals from and to which the electron is excited. As expected all of the transitions involve the $\pi$ system. Moreover, the natural transition orbitals show that the electron density is always transferred to the meso-position upon excitation (Figure S6), which supports an enhanced radiationless deactivation in the 8substituted compound 2 compared with $\mathbf{4}$ and $\mathbf{6}$. We also obtained the calculated emission spectra of $\mathbf{4}$ and $\mathbf{6}$ in toluene and methanol (Figure S7). Regardless of the computational approach overestimating the transition energies by approximately 2000 to $2500 \mathrm{~cm}^{-1}$, ${ }^{[9]}$ it successfully predicts an $\sim 85 \mathrm{~nm}$ bathochromic shift in the emission spectra of 6 with respect to 4 , perfectly matching the experimental $88 \pm 2 \mathrm{~nm}$ average difference between the emission maximum of the two dyes (averaged between the different solvents). The computational approach also predicts a bathochromic shift in the emission spectra of both dyes when passing from methanol to toluene, although the effect is less notable than that obtained experimentally. The calculated emission spectrum of $\mathbf{2}$ is very broad due to the flexible nature of the $S_{1}$ excited state geometry and would require several more timesteps than performed in the current study to resolve the band shape.

The dipole moments of the $\mathrm{S}_{0}$ and $\mathrm{S}_{1}$ excited-state geometries of each of the compounds are given in Table 2. For 4 and $\mathbf{6}$, an increase in the dipole moment of the $S_{1}$ state is observed upon relaxation of the geometry. Dye 2 exhibits a very solvent dependent $S_{1}$ dipole moment, corresponding to the different relaxed excited-state geometries seen in Figure 5.

Table 2. Calculated $S_{0}$ and $S_{1}$ dipole moments (in Debye) for each of the relaxed $S_{0}$ and $S_{1}$ geometries

\begin{tabular}{lllll} 
& \multicolumn{2}{c}{$\mathrm{S}_{0}$ relaxed geometry } & \multicolumn{2}{c}{$\mathrm{S}_{1}$ relaxed geometry } \\
BODIPY & $\mu\left(\mathrm{S}_{0}\right)$ & $\mu\left(\mathrm{S}_{1}\right)$ & $\mu\left(\mathrm{S}_{0}\right)$ & $\mu\left(\mathrm{S}_{1}\right)$ \\
\hline & Gas phase & & & \\
$\mathbf{2}$ & 8.52 & 7.13 & 8.04 & 3.18 \\
$\mathbf{4}$ & 4.12 & 2.54 & 3.82 & 2.65 \\
$\mathbf{6}$ & 1.74 & 1.66 & 1.52 & 2.14 \\
& Solvated (toluene) & & \\
\hline
\end{tabular}

Intracellular FLIM measurements.

The potentials of BODIPY $\mathbf{2 , 4}$, and $\mathbf{6}$ for use as biological labels and probes were demonstrated in live-cell FLIM microscopy.
Live breast cancer cells MDA-MB-231, one of the five clinical models of breast cancer, were cultured on glass slides, incubated with the BODIPY compounds 2, 4, and $\mathbf{6}$ and imaged in a confocal FLIM microscope. Figures 6 and S8 show representative examples of the collected images. The cells incorporated the dyes promptly, in less than 4-5 min. Typically, BODIPY dyes are neutral compounds that can penetrate cell membranes, and most of them tend to accumulate in subcellular membranes because of their relatively high lipophilicity. ${ }^{[27]}$ The modification of BODIPY with ionic substituents or more hydrophilic groups may increase the aqueous solubility, but will also decrease the ability to penetrate the cell membrane. ${ }^{[5]}$ In our case, the three compounds are neutral, and the images show an effective incorporation of the dyes into the cytosol and cellular organelles for compounds 2 and $\mathbf{4}$ (Figures $6 \mathrm{~A}$ and B). In contrast, compound $\mathbf{6}$, the most lipophilic dye, appears to be localized in membranes, endosomes and lysosomes. This is in agreement with the intracellular distribution of other BODIPY derivatives with styryl groups in their structures, ${ }^{[27 a]}$ which also accumulate in these organelles and the endoplasmic reticulum. The behaviour of 2 and $\mathbf{4}$ suggests that the presence of the benzylamine group provides sufficient hydrophilicity to be soluble in the cytosol, but these dyes retain the lipophilicity required to preserve cell membrane permeability. This balance could be the key for the use of these compounds in biological imaging. All three dyes maintained their emission levels after 1-2 $\mathrm{h}$ of collecting images in different regions of the slide, showing no signs of photobleaching or dye excretion/leakage from the cell. Likewise, there were no signs of morphological damage to the cells during the time of the experiments, which demonstrates the low toxicity caused by the dyes in such a time frame.
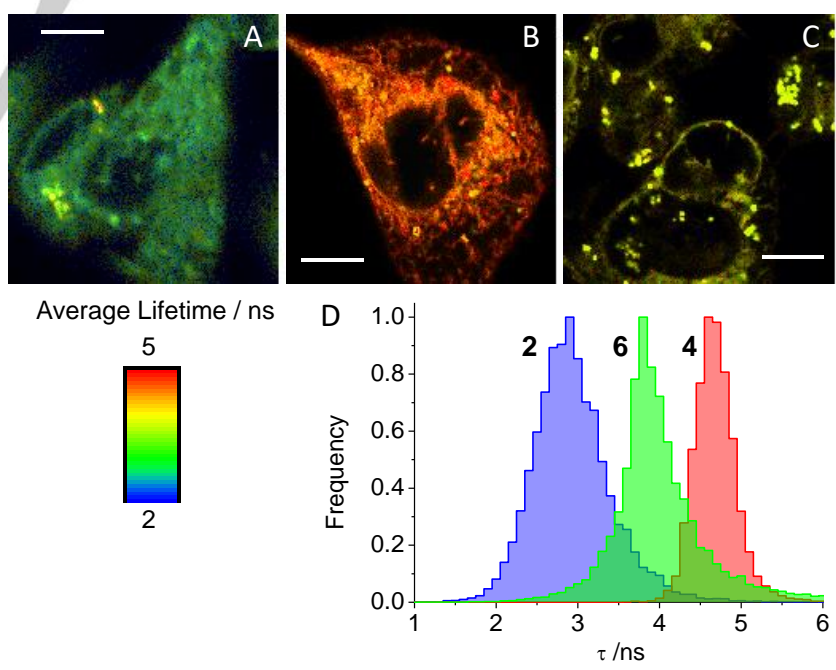

Figure 6. FLIM images of breast cancer cells MDA-MB-231 incubated with compound 2 (A), compound 4 (B) and compound 6 (C). (D) Lifetime distributions obtained from the pixels of interest within the images: compounds 2 (blue), 4 (red) and 6 (green). Scale bar represents $10 \mu \mathrm{m}$. 
Importantly, FLIM microscopy provides information on the different environments of the dyes when there are modifications in the fluorescence lifetime $\tau$, entailing an extra source of contrast for the images. The lifetime values collected from the analysis of the images exhibit normal distributions for all three dyes, with centres at 2.9 ns for compound 2, $4.6 \mathrm{~ns}$ for compound 4 , and $3.8 \mathrm{~ns}$ for compound $\mathbf{6}$. The values are in very good agreement with the values of the lifetime measured in the solvents tested (Tables 1, S3, S4 and S5). Perhaps, the most interesting compound in this sense is BODIPY 2, which presents a broader variation in its $\tau$ value with the solvent (Table S3). With the lifetime distribution centred at $2.9 \mathrm{~ns}$, the images suggest an environment close to 1-octanol, which may be considered a membrane model. In addition, by adjusting the contrast scale of the FLIM images, our results evidence differences in the environment of dyes 2 and 4 (Figure S9). In both cases, bright vesicular regions are found, in which 2 exhibits a larger lifetime, whereas $\mathbf{4}$ shows a shorter lifetime. For both dyes, these lifetime features are in agreement with a more hydrophobic environment. Likewise, the structure of BODIPY 2 is similar to other BODIPY dyes that have been used to probe environmental viscosity through changes in the fluorescence lifetime, thanks to the hindered rotation of the substituent at the meso-position in viscous media. These viscosity probes, BODIPY rotors, have been successfully employed to examine DNA interactions ${ }^{[7 c]}$ and report intracellular viscosity in live cell organelles and plasma membranes ${ }^{[7 a, 7 b]}$ using FLIM microscopy. As the Catalán analyses did not show any trends of the radiative and radiationless rate constants with the solvent parameters, other solvent features may be involved. Solvent viscosity is a physical property that may also affect the excited-state deactivation, and therefore, contribute to the contrast in FLIM microscopy; although other important factors, such as temperature ${ }^{[28]}$ or interactions with proteins, ${ }^{[29]}$ may also affect the lifetime of BODIPY rotors. In any case, 2 and $\mathbf{4}$ permit simultaneous imaging of different organelles, just by adding lifetime contrasts.

\section{Conclusions}

We have reported herein the synthesis of two new 3benzylaminoBODIPYs with emission in the green spectral region and one 3-benzylaminoBODIPY compound in the red emission region. We have described their photophysical properties, including the systematic analysis of the solvatochromism based on the Catalán methodology, and compared them with a previously published 8-benzylaminoBODIPY and other phenylamino-modified BODIPY dyes. We have found that solvent polarizability is the main property that governs the spectral maxima and spectral shifts of the 3-benzylaminesubstituted dyes among the different solvents.

Finally, we have tested the feasibility of these dyes for their use in cellular imaging. Thanks to the presence of the benzylamine group, dyes 2 and $\mathbf{4}$ exhibited fast membrane penetration, organelle staining, and added environment contrast in FLIM microscopy. In contrast, compound $\mathbf{6}$ mainly accumulated in endosomes because of its enhanced hydrophobicity.

This work continues the evaluation of the rational design of BODIPY dyes for the development of tailored probes with controllable properties that are useful in cellular imaging.

\section{Experimental Section}

\section{Spectroscopic and photophysical data}

Absorption spectra and spectrally corrected steady-state fluorescence emission spectra were collected using a Perkin-Elmer Lambda 650 UV/Vis spectrophotometer and a JASCO FP-8500 spectrofluorometer, respectively. All spectroscopic measurements were carried out in a Peltier temperature-controlled cell holder set at $20^{\circ} \mathrm{C}$, using undegassed samples in $5 \times 10 \mathrm{~mm}$ cuvettes (with $10 \mathrm{~mm}$ optical path length for absorption and $\mathrm{a} 90^{\circ}$ angle setup for fluorescence collection through the shortest side). Fluorescence quantum yield $(\Phi)$ determination was performed according to eqn. (1) ${ }^{[30]}$

$\Phi_{x}=\Phi_{r} \times \frac{F_{x}}{F_{r}} \times \frac{1-10^{-A_{r}\left(A_{e x}\right)}}{1-10^{-A_{x}\left(A_{e x}\right)}} \times \frac{n_{x}^{2}}{n_{r}^{2}}$

where $x$ and $r$ refer respectively to sample $x$ (i.e., the BODIPY derivative in a specific solvent) and the reference (standard) fluorophore with known quantum yield $\left(\Phi_{r}\right) ; F$ denotes the integrated fluorescence spectra, with spectral and inner-filter correction (the latter was minimized by keeping the absorbance below 0.1 , but in cases of small Stokes shifts, the correction was needed); $A\left(\lambda_{\text {ex }}\right)$ stands for the absorbance at the corresponding excitation wavelength; and $n$ is the refractive index of the solvent. Fluorescein in $0.1 \mathrm{~N} \mathrm{NaOH}$ was employed as the fluorescence quantum yield reference $\left(\Phi_{r}=0.91\right)$ for BODIPYs 4 and 8; and rhodamine 101 in ethanol $\left(\Phi_{r}=0.96\right)$ as a reference for $6{ }^{[31]}$ For each quantum yield determination, eight independent $\Phi_{\mathrm{x}}$ measurements were performed, using (2 conc. of sample $x) \times(2$ conc. of reference $r) \times(2$ excitation wavelengths, $\left.\lambda_{\text {ex }}\right)$.

Time-resolved fluorescence decay traces were recorded using a FluoTime200 fluorometer (PicoQuant $\mathrm{GmbH}$ ) working in single-photon timing mode. ${ }^{[32]}$ The pulsed excitation source was a $485 \mathrm{~nm}$ or a $532 \mathrm{~nm}$ diode laser ( $\mathrm{LDH}$ series from PicoQuant $\mathrm{GmbH}$ ) for compounds $\mathbf{4}$ and $\mathbf{6}$, respectively, both operated with a PDL-800 driver (PicoQuant) at a pulse repetition rate of $20 \mathrm{MHz}$. For each compound in a given solvent, four different fluorescence decay traces were collected at different emission wavelengths, selected by a grating monochromator, after a polarizer set at the 'magic angle'. These emission wavelengths were 520, 530, 540, and $550 \mathrm{~nm}$ for 4 ; and $605,610,615$, and $620 \mathrm{~nm}$ for 6 . The fluorescence decay traces were collected over 1320 channels, with a time increment of $36 \mathrm{ps}$ per channel, until they reached $2 \times 10^{4}$ counts in the peak channel. Histograms of the instrument response functions were collected using a LUDOX scatterer. Fluorescence lifetimes $(\tau)$ were obtained from the iterative deconvolution fitting of the decay trace convoluted with the instrumental response function. In all cases, the fluorescence decay histograms collected at four different emission wavelengths could be fitted globally with a single exponential function.

\section{Quantum-chemical calculations}

Density functional theory (DFT) was used with the wB97X-D functional and $6-31 G(d)$ basis set. Geometries of the $S_{0}$ ground state were optimized, along with the geometries of the $S_{1}$ excited states (using timedependent DFT). Solvated fluorescence emission energies were calculated using the polarisable continuum model (PCM), with the following dielectric constants: $\mathrm{MeOH}$ (32.7) and toluene (2.38). Simulated 
emission spectra were generated using ab initio molecular dynamics on the $S_{1}$ potential energy surface (TDDFT). The temperature was set to $298 \mathrm{~K}$, with a timestep of 25 au and a total of 200 timesteps. Initial velocities were sampled using the Maxwell-Boltzmann distribution. Natural transition orbitals (NTO) were calculated using the methodology set out in reference ${ }^{[25]}$. All calculations were performed with the Q-Chem 5.0 software.

\section{Fluorescence Lifetime Imaging Microscopy (FLIM)}

FLIM images were recorded with a MicroTime 200 system (PicoQuant, $\mathrm{GmbH}$, Germany) based on single-photon timing with the time-tagged time-resolved (TTTR) methodology, which permits the reconstruction of the fluorescence decay traces from the BODIPY dyes in the confocal volume. Different pulsed diode lasers were employed as excitation sources (all from the LDH series from PicoQuant), depending on the dye to be imaged. Table S6 gathers the instrumental settings for each dye. Both the $375 \mathrm{~nm}$ and $470 \mathrm{~nm}$ laser heads were controlled with a Sepia II driver (PicoQuant), whereas the $532 \mathrm{~nm}$ laser was controlled with a PDL800 driver (PicoQuant), all of them set at a repetition rate of $20 \mathrm{MHz}$. The corresponding excitation laser beam was directed into the specimen through an apochromatic oil immersion objective $(100 \times 1.4 \mathrm{NA})$ of an inverted microscope system (IX-71, Olympus, Japan). The fluorescence light was collected back and filtered by the main dichroic and cut-off filters, and spatially filtered through a $75 \mu \mathrm{m}$ pinhole. The fluorescence photons were detected by an avalanche photodiode (SPCM-AQR-14, Perkin Elmer), after passing through the adequate bandpass filter. Photon counting and time tagging were performed within a TimeHarp 200 module (PicoQuant), with a time resolution of 29 ps per channel. To image a region, the sample was raster-scanned with an $x-y$ piezo-driven device (Physik Instrumente, Germany). The image data were normally acquired over a surface area between 1592 and $4890 \mu \mathrm{m}^{2}$, with a $512 \times$ 512 pixel resolution and a collection time of $0.60 \mathrm{~ms}$ per pixel.

The FLIM images were analysed using SymphoTime 32 software (PicoQuant). To obtain the fitted FLIM images, a reconstructed instrument response function was employed to analyse the fluorescence decay histogram of each pixel within the whole image using a monoexponential model. The decay time was a freely adjustable parameter. The decay histograms were fitted by applying the maximum likelihood estimator (MLE), which yields the correct parameter sets for low count rates. ${ }^{[33]} \mathrm{A}$ previous spatial binning of $3 \times 3$ pixels and prehistogramming of 8 temporal channels (for a final resolution of 232 ps/channel) were used to achieve a larger number of counts in each pixel.

\section{Cell culture}

The Cell Culture Facility, Genyo, Pfizer-University of Granada-Junta de Andalucia centre for genomics and oncological research, provided the MDA-MB-231 cell line. Cells were grown in Dulbecco's Modified Eagle Medium (DMEM) supplemented with 10\% (v/v) Foetal Bovine Serum (FBS), $2 \mathrm{mM}$ glutamine, $100 \mathrm{U} / \mathrm{mL}$ penicillin, and $0.1 \mu \mathrm{g} / \mathrm{mL}$ streptomycin at $37 \stackrel{\circ}{\circ} \mathrm{C}$ in a humidified $5 \% \mathrm{CO}_{2}$ incubator. For the FLIM experiments, MDA-MB-231 cells were seeded onto 20-mm-diameter glass slides at a density of $11250 \mathrm{cells} / \mathrm{cm}^{2}$. The glass slides were washed with the DMEM and phosphate-buffered saline (PBS) before adding the cells. Before adding the dyes, the DMEM was removed and exchanged with 1 $\mathrm{mL}$ of PBS buffer at $\mathrm{pH}$ 8. The different BODIPY dyes were added directly to the cells seeded onto the glass slide in a concentration of $1 \times$ $10^{-7} \mathrm{M}$. The cells were washed twice with PBS buffer at $\mathrm{pH} 8$. All dyes passively entered into the cell in 4-5 minutes.

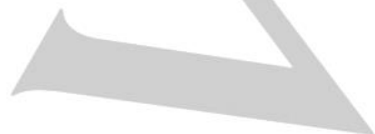

\section{Acknowledgements}

This work has been financially supported by the National Nature Science Foundation of China (Grants Nos. 21672006) and Nature Science Foundation of Anhui Province (Grants No 1508085J07); and grant CTQ2014-56370-R from the Spanish Ministry of Economy and Competitiveness and the European Regional Development Fund (ERDF).

\section{Keywords: Fluorescent probes • FLIM microscopy •} Solvatochromism

[1] a) R. P. Haugland, The Molecular Probes $®$ Handbook—A Guide to Fluorescent Probes and Labeling Technologies, 11th ed., Life Technologies Corp. , Eugene OR (USA), 2010; b) A. Loudet, K. Burgess, Chem. Rev. 2007, 107, 4891-4932; c) G. Ulrich, R. Ziessel, A Harriman, Angew. Chem. Int. Ed. 2008, 47, 1184-1201; d) N. Boens, V. Leen, W. Dehaen, Chem. Soc. Rev. 2012, 41, 1130-1172.

[2] N. Boens, B. Verbelen, W. Dehaen, Eur. J. Org. Chem. 2015, 2015, 6577-6595.

[3] a) N. J. Findlay, J. Bruckbauer, A. R. Inigo, B. Breig, S. Arumugam, D. J. Wallis, R. W. Martin, P. J. Skabara, Adv. Mater. 2014, 26, 7290-7294; b) A. Zampetti, A. Minotto, B. M. Squeo, V. G. Gregoriou, S. Allard, U. Scherf, C. L. Chochos, F. Cacialli, Sci. Rep. 2017, 7, 1611; c) C. L. Liu, Y. Chen, D. P. Shelar, C. Li, G. Cheng, W. F. Fu, J Mater Chem C 2014 2, 5471-5478

[4] a) H. Klfout, A. Stewart, M. Elkhalifa, H. S. He, Acs App/ Mater Inter 2017, 9, 39873-39889; b) J. X. Liao, H. B. Zhao, Y. J. Xu, W. N. Zhou, F. Peng, Y. Wang, Y. T. Fang, Rsc Advances 2017, 7, 33975-33985.

[5] T. Kowada, H. Maeda, K. Kikuchi, Chem. Soc. Rev. 2015, 44, 49534972.

[6] M. Ruedas-Rama, J. Alvarez-Pez, L. Crovetto, J. Paredes, A. Orte, in Advanced Photon Counting, Vol. 15 (Eds.: P. Kapusta, M. Wahl, R. Erdmann), Springer International Publishing, 2015, pp. 191-223.

[7] a) P. S. Sherin, I. Lopez-Duarte, M. R. Dent, M. Kubankova, A. Vysniauskas, J. A. Bull, E. S. Reshetnikova, A. S. Klymchenko, Y. P. Tsentalovich, M. K. Kuimova, Chem. Sci. 2017, 8, 3523-3528; b) D. D. Su, C. L. Teoh, N. Y. Gao, Q. H. Xu, Y. T. Chang, Sensors 2016, 16; c) D. Dziuba, P. Jurkiewicz, M. Cebecauer, M. Hof, M. Hocek, Angew. Chem. Int. Ed. 2016, 55, 174-178.

[8] a) C. A. Osorio-Martínez, A. Urías-Benavides, C. F. A. Gómez-Durán, J. Bañuelos, I. Esnal, I. López Arbeloa, E. Peña-Cabrera, J. Org. Chem. 2012, 77, 5434-5438; b) V. Leen, P. Yuan, L. Wang, N. Boens, W. Dehaen, Org. Lett. 2012, 14, 6150-6153; c) B. Dhokale, T. Jadhav, S. M. Mobin, R. Misra, Dalton Trans. 2015, 44, 15803-15812; d) A. Orte, E. Debroye, M. J. Ruedas-Rama, E. Garcia-Fernandez, D. Robinson, L. Crovetto, E. M. Talavera, J. M. Alvarez-Pez, V. Leen, B. Verbelen, L. C. D. de Rezende, W. Dehaen, J. Hofkens, M. Van der Auweraer, N Boens, Rsc Advances 2016, 6, 102899-102913.

[9] N. Boens, L. Wang, V. Leen, P. Yuan, B. Verbelen, W. Dehaen, M. Van der Auweraer, W. D. De Borggraeve, L. Van Meervelt, J. Jacobs, D. Beljonne, C. Tonnelé, R. Lazzaroni, M. J. Ruedas-Rama, A. Orte, L. Crovetto, E. M. Talavera, J. M. Alvarez-Pez, J. Phys. Chem. A 2014, 118, 1576-1594.

[10] a) L. Y. Zhang, J. C. Er, H. Jiang, X. Li, Z. F. Luo, T. Ramezani, Y. Feng, M. K. Tang, Y. T. Chang, M. Vendrell, Chemical Communications 2016, 52, 9093-9096; b) W. W. Qin, V. Leen, T. Rohand, W. Dehaen, P. Dedecker, M. Van der Auweraer, K. Robeyns, L. Van Meervelt, D. Beljonne, B. Van Averbeke, J. N. Clifford, K. Driesen, K. Binnemans, N. Boens, J Phys Chem A 2009, 113, 439-447.

[11] Y. Ni, J. Wu, Org. Biomol. Chem. 2014, 12, 3774-3791.

[12] a) T. Rohand, M. Baruah, W. W. Qin, N. Boens, W. Dehaen, Chem. Commun. 2006, 266-268; b) M. Baruah, W. W. Qin, N. Basaric, W. M. De Borggraeve, N. Boens, J Org Chem 2005, 70, 4152-4157; c) T. Jiang, P. Zhang, C. J. Yu, J. Yin, L. J. Jiao, E. Dai, J. Wang, Y. Wei, X L. Mu, E. H. Hao, Org. Lett. 2014, 16, 1952-1955.

[13] X. Zhou, C. J. Yu, Z. Y. Feng, Y. Yu, J. Wang, E. H. Hao, Y. Wei, X. L. $\mathrm{Mu}$, L. J. Jiao, Org. Lett. 2015, 17, 4632-4635.

[14] L. J. Jiao, W. D. Pang, J. Y. Zhou, Y. Wei, X. L. Mu, G. F. Bai, E. H. Hao, J Org Chem 2011, 76, 9988-9996.

[15] W. Zhou, M. G. Fan, J. L. Yin, Y. W. Jiang, D. W. Ma, J. Am. Chem Soc. 2015, 137, 11942-11945.

[16] I. J. Arroyo, R. Hu, G. Merino, B. Z. Tang, E. Peña-Cabrera, J. Org. Chem. 2009, 74, 5719-5722. 
[17] W. W. Qin, M. Baruah, M. Sliwa, M. Van der Auweraer, W. M. De Borggraeve, D. Beljonne, B. Van Averbeke, N. Boens, J Phys Chem A 2008, 112, 6104-6114.

[18] T. V. Goud, A. Tutar, J. F. Biellmann, Tetrahedron 2006, 62, 5084-5091.

[19] W. Qin, M. Baruah, M. Van der Auweraer, F. C. De Schryver, N. Boens, J. Phys. Chem. A 2005, 109, 7371-7384.

[20] a) T. Rohand, W. W. Qin, N. Boens, W. Dehaen, Eur. J. Org. Chem 2006, 4658-4663; b) M. Baruah, W. W. Qin, C. Flors, J. Hofkens, R. A L. Vallee, D. Beljonne, M. Van der Auweraer, W. M. De Borggraeve, N. Boens, J Phys Chem A 2006, 110, 5998-6009.

[21] W. W. Qin, V. Leen, W. Dehaen, J. Cui, C. Xu, X. L. Tang, W. S. Liu, T. Rohand, D. Beljonne, B. Van Averbeke, J. N. Clifford, K. Driesen, K. Binnemans, M. Van der Auweraer, N. Boens, J Phys Chem C 2009, 113, 11731-11740.

[22] J. Catalán, J. Phys. Chem. B 2009, 113, 5951-5960.

[23] D. Pevenage, D. Corens, W. Dehaen, M. Van der Auweraer, F. C. De Schryver, B Soc Chim Belg 1997, 106, 565-572.

[24] E. A. Briggs, N. A. Besley, D. Robinson, J. Phys. Chem. A 2013, 117, 2644-2650.

[25] L. Jiao, C. Yu, J. Wang, E. A. Briggs, N. A. Besley, D. Robinson, M. J. Ruedas-Rama, A. Orte, L. Crovetto, E. M. Talavera, J. M. Alvarez-Pez, M. Van der Auweraer, N. Boens, RSC Adv. 2015, 5, 89375-89388.

[26] I. Mayer, Chem. Phys. Lett. 2007, 437, 284-286.

[27] a) Q. D. Zheng, G. X. Xu, P. N. Prasad, Chem-Eur J 2008, 14, 58125819; b) L. J. Jiao, C. J. Yu, T. Uppal, M. M. Liu, Y. Li, Y. Y. Zhou, E. H.
Hao, X. K. Hu, M. G. H. Vicente, Org. Biomol. Chem. 2010, 8, 25172519.

[28] A. Vysniauskas, I. Lopez-Duarte, N. Duchemin, T. T. Vu, Y. L. Wu, E. M. Budynina, Y. A. Volkova, E. P. Cabrera, D. E. Ramirez-Ornelas, M. K. Kuimova, Phys. Chem. Chem. Phys. 2017, 19, 25252-25259.

[29] C. Yu, W. Miao, J. Wang, E. Hao, L. Jiao, ACS Omega 2017, 2, 35513561.

[30] a) B. Valeur, M. N. Berberan-Santos, Molecular Fluorescence. Principles and Applications. , $2^{\text {nd }}$ edn. ed., Wiley-VCH Verlag $\mathrm{GmbH}$, Weinheim (Germany), 2012; b) J. R. Lakowicz, Principles of Fluorescence Spectroscopy, 3rd ed., Springer, 2006.

[31] C. Wurth, M. Grabolle, J. Pauli, M. Spieles, U. Resch-Genger, Nat Protoc 2013, 8, 1535-1550.

[32] a) M. vandeVen, M. Ameloot, B. Valeur, N. Boens, J. Fluoresc. 2005 , 15, 377-413; b) H. Lemmetyinen, N. V. Tkachenko, B. Valeur, J.-i. Hotta, M. Ameloot, N. P. Ernsting, T. Gustavsson, N. Boens, Pure Appl. Chem. 2014, 86, 1969-1998; c) W. Becker, Advanced Time-Correlated Single Photon Counting Techniques, Vol. 81, Springer, Berlin (Germany), 2005.

[33] M. Maus, M. Cotlet, J. Hofkens, T. Gensch, F. C. De Schryver, J. Schaffer, C. A. M. Seidel, Anal. Chem. 2001, 73, 2078-2086. 
Entry for the Table of Contents (Please choose one layout)

Layout 1:

\section{FULL PAPER}

New BODIPY dyes substituted with a benzylamino group have been synthesized and characterized. The position of the benzylamino group has shown a significant influence on the spectroscopic and photophysical properties, which have been explored in different solvents. The dyes have been employed as probes in the fluorescence lifetime imaging of living cells, demonstrating their high potential for bioimaging.

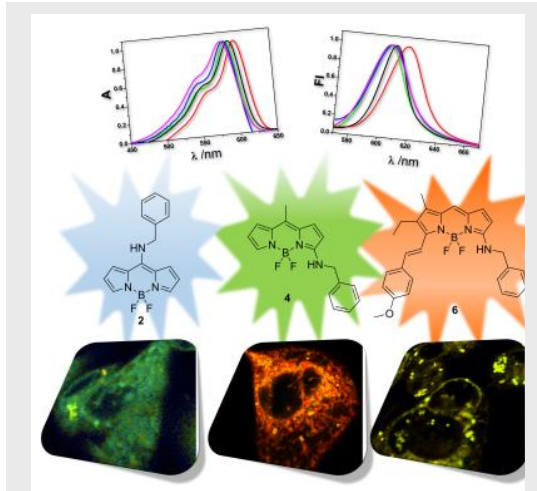

*one or two words that highlight the emphasis of the paper or the field of the study
Key Topic* BODIPY bioimaging

Consuelo Ripoll, Cheng Cheng, Emilio Garcia-Fernandez, Jin Li, Angel Orte, Hainam Do, Lijuan Jiao, * David Robinson, * Luis Crovetto, Juan A. González-Vera, Eva M. Talavera, Jose M. Alvarez-Pez, Noël Boens, ${ }^{*}$ and María Jose Ruedas-Rama*

Page No. - Page No.

Synthesis and spectroscopy of benzylamine-substituted BODIPYs for bioimaging 